\title{
Effect of improving dietary quality of food parcels on dietary intake in Dutch food bank recipients - a randomized controlled trial
}

\author{
Judith Neter $^{1}$, Coosje Dijkstra ${ }^{1}$, Jos Twisk ${ }^{2}$, Marjolein Visser ${ }^{1}$ and Ingeborg Brouwer ${ }^{1}$ \\ ${ }^{1}$ Department of Health Sciences, Faculty of Science, Vrije Universiteit Amsterdam, Amsterdam Public Health Research \\ Institute, Amsterdam, Netherlands and \\ ${ }^{2}$ Department of Epidemiology and Biostatistics, Amsterdam UMC, Location VU University Medical Center, \\ Amsterdam Public Health Research Institute, Amsterdam, Netherlands
}

\section{Abstract}

Introduction: Since food banks have a strong influence on recipients' diets, and seem to have difficulties in supporting healthy diets, improving the dietary quality of food parcels is important. Therefore, we aimed to assess whether improving the dietary quality of food parcels, using different strategies, can positively impact the actual dietary intake of Dutch food bank recipients.

Materials and methods: This randomized cross-over controlled trial with four intervention conditions [1) Control (standard food parcel), 2) Snacks ${ }^{-}$(standard food parcel with replacement of snacks by staple foods), 3) $\mathrm{FV}^{+}$(standard food parcel plus the recommended daily amount of fruit and vegetables), 4) Snacks ${ }^{-}+$FV (standard food parcel with replacement of snacks by staple foods plus the recommended daily amount of fruit and vegetables)] included food bank recipients from three food banks. In total, 199 recipients were randomly allocated. At baseline, participants filled in a questionnaire and underwent anthropometric measurements. Dietary intake data were collected through 24-hour recalls after both intervention conditions at 4 and 8 weeks follow-up. Primary outcome was fruit and vegetable intake, secondary outcomes were dietary intakes of food groups and nutrients.

Results: Multi-level linear regression analysis, using a two-level model showed a higher mean fruit intake in participants in the FV ${ }^{+}$condition than in participants in the Control condition $(\delta: 74[40.3 ; 107.6] \mathrm{g})$. Both mean fruit and mean vegetable intake were higher in participants in the Snacks ${ }^{-}+\mathrm{FV}^{+}$condition than in participants in the Control condition (fruit $\delta$ : 81.3 [56.5;106.2] g; vegetable: $\delta: 46.2[17.5 ; 74.9] \mathrm{g}$ ), as well as in the Snacks ${ }^{-}$condition (fruit: $\delta: 70.0$ [38.8;101.1] g; vegetable $\delta: 62.2$ [26.2; 98.2$] \mathrm{g}$ ).

Discussion: This study shows that improving the dietary content of food parcels can positively impact the dietary intake of Dutch food bank recipients. With this we can further develop effective strategies to improve dietary intake of food bank recipients.

\section{Conflict of Interest}

There is no conflict of interest 